\title{
Pulmonary embolism with cardiac arrest: a STEMI patient's unexpected course
}

\author{
Zoë Piggott, BAH, MD*; Tomislav Jelic, BSc, MD*
}

\begin{abstract}
We describe the successful use and complications of bolus-dose alteplase to treat strongly suspected pulmonary embolism (PE) with cardiac arrest in a patient initially presenting as ST-elevation myocardial infarcation (MI). Case description is followed by a review of the indications, safety, and dosing of systemic thrombolytic therapy for highrisk PE in the emergency department (ED). Diagnostic and therapeutic approach to PE in critically ill patients is also considered, including the potential utility of point-of-care ultrasound (PoCUS) in the ED.
\end{abstract}

\section{RÉSUMÉ}

Nous ferons état, dans l'article, de l'administration fructueuse d'altéplase en bolus et de ses complications dans un cas de forte présomption d'embolie pulmonaire (EP) suivie d'un arrêt cardiaque, qui s'est manifestée au début comme un infarctus du myocarde (MI) avec élévation du segment ST. Suivra un examen des indications, de l'innocuité et de la posologie du traitement thrombolytique par voie générale dans les cas d'EP à risque élevé au service des urgences (SU). Il sera également question de l'approche diagnostique et thérapeutique de l'EP chez les patients se trouvant dans un état très grave, y compris de l'utilité possible de l'échographie au lieu d'intervention, au SU.

Keywords: pulmonary embolism, cardiac arrest, thrombolysis, STEMI

\section{CASE REPORT}

We present the case of a previously well 41-year-old male who presented to our emergency department (ED) after a witnessed syncopal episode. No further history was immediately available. A pre-hospital echocardiogram (ECG) showed ST-segment elevation in leads V1-V3 (Figure 1). Consequently, the patient was accepted to the ST-elevation myocardial infarction (STEMI) ED bypass protocol by our hospital and was being transported for immediate coronary angiography.

The patient's clinical condition deteriorated en route, and attending paramedics elected to deliver the patient to the ED instead. Upon arrival at 16:40, the patient was cyanotic, dyspneic, and somnolent. Central pulses were palpable, although non-invasive blood pressure and oxygen saturation were undetectable. The skin of his lower extremities was mottled. Engorged superficial veins were noted across the patient's abdomen and right flank; examination of the lower extremities revealed clusters of engorged varicose and superficial veins over the medial right thigh. Supplemental oxygen, intravenous (IV) normal saline, and norepinephrine infusion were initiated. His ED ECG showed lateral ST depression, ST elevation in aVR and $\mathrm{V} 1$, and an incomplete right bundle branch block pattern (Figure 2). Bedside point-of-care ultrasound (PoCUS), performed in the resuscitation room, revealed a hyperdynamic and partially collapsed left ventricle, dilated right ventricle, and global right ventricular systolic dysfunction (Video 1). A pulmonary embolus (PE) was strongly suspected.

The patient's level of consciousness continued to deteriorate during his initial assessment and resuscitation. At 16:47, uncomplicated rapid sequence intubation was

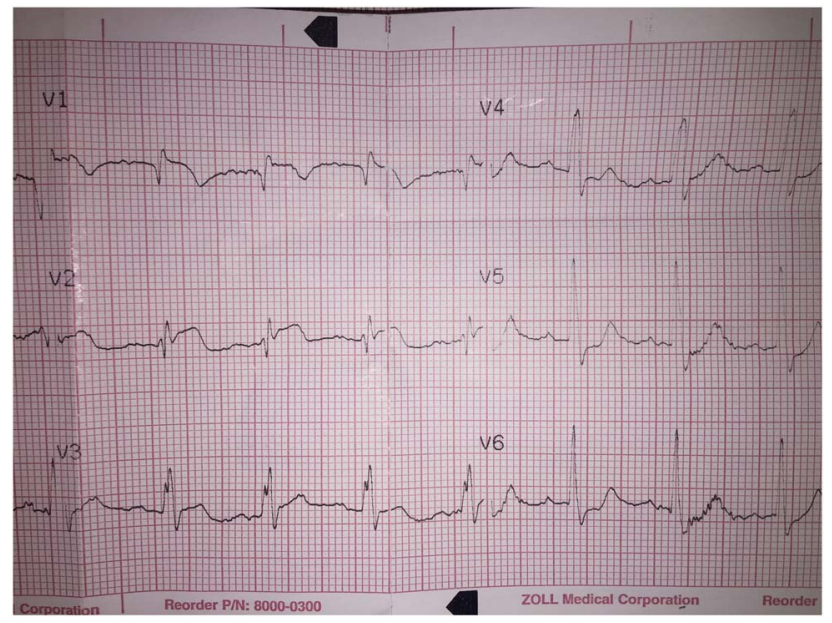

Figure 1. Pre-hospital ECG strip, initially interpreted as anterior ST-elevation MI by our hospital's STEMI bypass program.

From the *Department of Emergency Medicine, University of Manitoba, Winnipeg, MB.

Correspondence to: Dr. Zoë Piggott, Room L1019, St. Boniface Hospital, Winnipeg, MB R2H 2A6; Email: zoepiggott@gmail.com 


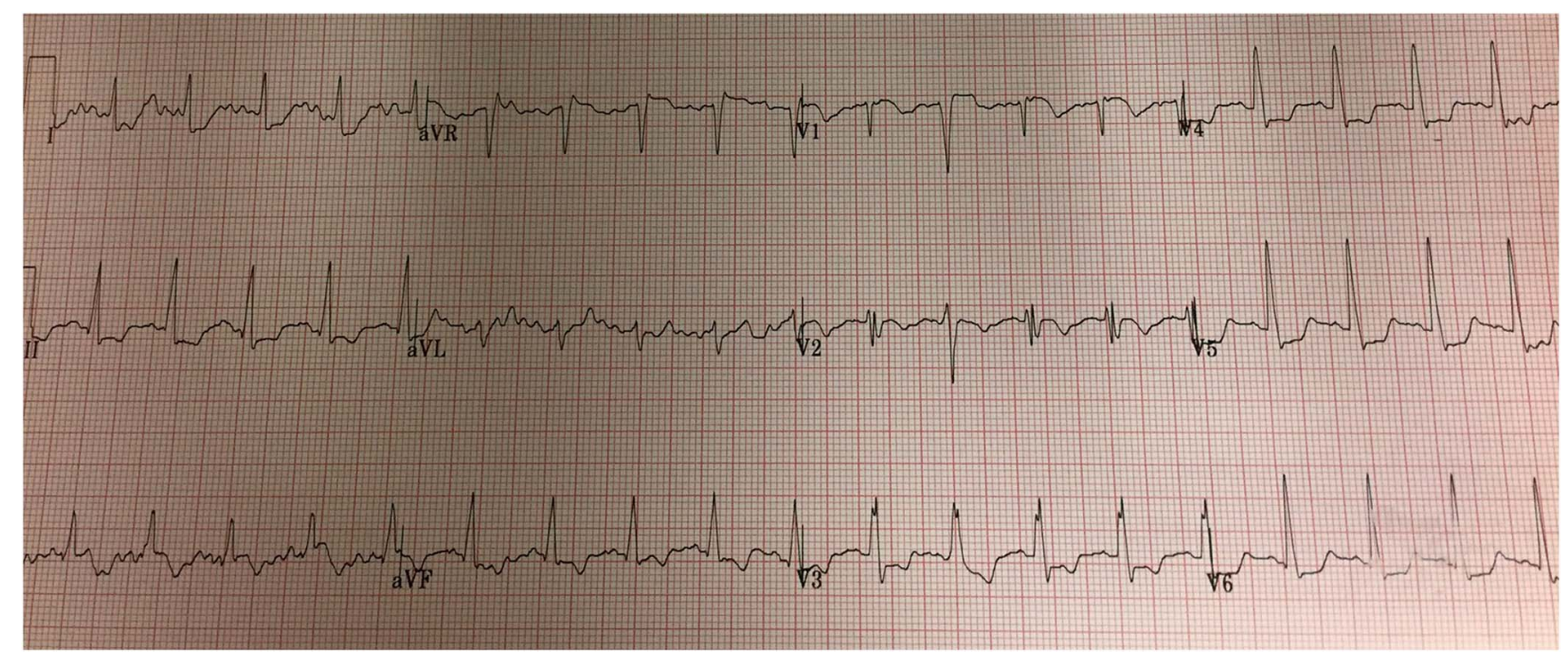

Figure 2. Emergency department 12-lead ECG. Findings include sinus tachycardia, lateral ST depression, ST elevation in $\mathrm{aVR}$ and $\mathrm{V} 1$, and incomplete RBBB. The QRS duration is $100 \mathrm{~ms}$.

Video 1. Bedside PoCUS video of our patient, demonstrating RV dilation and systolic dysfunction consistent with PE.

performed using $1 \mathrm{mg} / \mathrm{kg}$ of IV ketamine and $1 \mathrm{mg} / \mathrm{kg}$ of IV rocuronium. One hundred milligrams of IV alteplase (tPA) was initiated at 16:53; the first 10\% was ordered as a bolus, with the remainder infused over one hour. However, the patient experienced a witnessed pulseless electrical activity (PEA) arrest at 16:56. Cardiopulmonary resuscitation (CPR) and standard advanced cardiac life support (ACLS) were initiated immediately, and a total $100 \mathrm{mg}$ of tPA was deliberately infused as a rapid bolus over the next two minutes. After four minutes of CPR, the return of spontaneous circulation (ROSC) was achieved with a blood pressure of $138 / 90$ and heart rate of 109 . At 17:23, venous blood gas results were as follows: $\mathrm{pH} 6.97, \mathrm{pCO}_{2} 84, \mathrm{pO}_{2} 28$, $\mathrm{HCO}_{3}$ 12, and lactate 10.2. A portable chest X-ray confirmed clear lung fields bilaterally. An IV heparin bolus was initiated, and the patient was admitted to the intensive care unit (ICU).

After his resuscitation and admission to the hospital, additional medical history became available. Prior magnetic resonance imaging (MRI) demonstrated abnormally large and tortuous saphenous and perforator veins extending the length of the right thigh. The patient had experienced several episodes of right-sided superficial thrombophlebitis, but no prior deep venous thromboembolic (DVT) disease. He had been horseback riding a few days before his presentation, with subsequent shortness of breath and new right thigh discomfort.

Pressor support was successfully weaned overnight, and he was extubated without incident the following morning. That afternoon, the signs and symptoms of hemorrhagic shock were noted, and a large hemoperitoneum, likely from a splenic artery, was detected on abdominal computed tomography (CT). Parenteral anticoagulation was stopped, and five units of packed red cells were transfused. The patient was transferred to the interventional radiology suite, where he underwent successful endovascular coiling of the bleeding splenic vessels and insertion of an inferior vena cava (IVC) filter. On admission day six, the diagnosis of bilateral $\mathrm{PE}$ was confirmed by CT pulmonary angiography, which showed clots in the left main pulmonary artery, right upper segmental pulmonary artery, and multiple 
other smaller vessels. He was discharged from the hospital with no noted neurologic or functional deficits.

Two years after his presentation, our patient has no functional limitations or symptoms of post-thrombotic syndrome and has returned to full-time work in his highly active profession. His IVC filter has been removed, and anticoagulation maintained with rivaroxaban.

\section{DISCUSSION}

\section{Diagnosis of high-risk PE}

$\mathrm{PE}$ is the third most common cause of cardiovascular death and carries a high mortality rate when presenting with hemodynamic instability or cardiac arrest (52\%$65 \%) .{ }^{1-4}$ It may account for up to $15 \%$ of in-hospital deaths and $2 \%$ of out-of-hospital cardiac arrests. ${ }^{1}$

The definition of high-risk PE proposed by the European Society of Cardiology and American Heart Association is widely accepted: PE with hemodynamic instability, i.e., signs of shock requiring inotropic support, documented systolic blood pressure $<90 \mathrm{~mm} \mathrm{Hg}$ for $>15$ minutes, or both. ${ }^{5-8}$

Definitive diagnosis of $\mathrm{PE}$ in critically ill and peri-arrest ED patients remains a significant challenge. In many centres, D-dimer results may not be available within a clinically appropriate time frame. In patients who arrested, elevated D-dimer levels are also associated with other critical illnesses and systemic endothelial injury. ${ }^{9}$ Most are too unwell for transfer to the radiology suite for imaging. Furthermore, clinicians may not always consider the diagnosis of PE if confronted with a peri-arrest patient, particularly if the history and collateral information are limited. Therefore, the clinician must rely on select historical points, physical exam findings, and bedside diagnostic tests:

1. History: prior DVT or PE; clotting disorder; active cancer; and recent immobilization, airline travel, or orthopedic surgery. ${ }^{10}$

2. Physical exam: unilateral leg swelling; oxygen saturation $<95 \%$; witnessed cardiac arrest; and initial arrest rhythm PEA (particularly with narrow QRS). ${ }^{10}$

3. ECG: precordial T-wave inversion; precordial ST depression; precordial Qr pattern; S1Q3T3; new right bundle branch block; right or indeterminate axis deviation; and ST elevation in aVR. ${ }^{11,12}$

4. PoCUS: right ventricular dilation, hypokinesis, or both; and non-compressible popliteal, femoral veins (suggesting concomitant DVT), or both. ${ }^{13,14}$

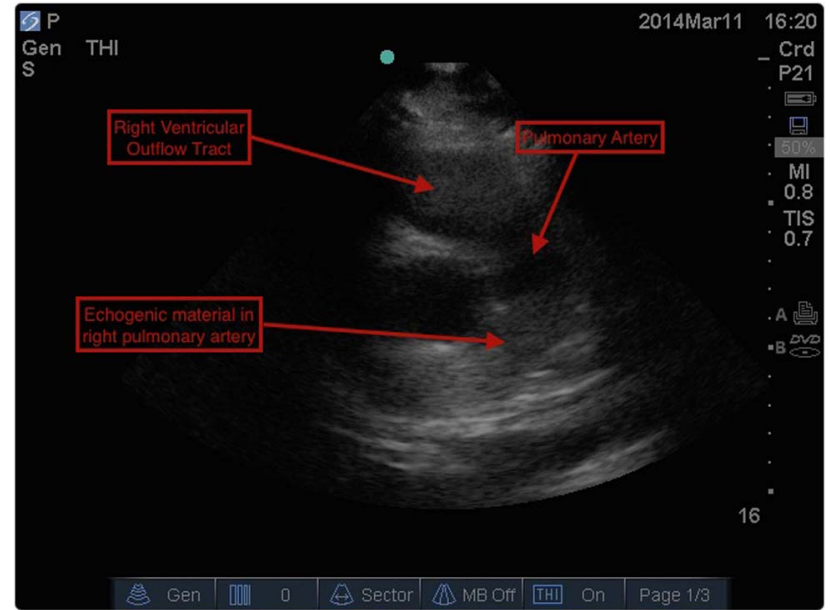

Figure 3. PoCUS still image showing echogenic material, suspicious for clot, in the patient's right pulmonary artery.

Anterior ST elevation, noted in our patient's prehospital ECG, is uncommon in acute PE, though it has been described. ${ }^{11,15,16}$ Proposed mechanisms include right ventricular strain and injury because of increased afterload; rarely, this finding can reflect coronary occlusion due to a clot through a patent foramen ovale. ${ }^{15,17}$ It is often visually indistinguishable from the ST elevation seen in an acute myocardial infarction; however, the simultaneous (or subsequent) presence of more specific ECG findings may raise the index of suspicion for $\mathrm{PE}$ at a patient's bedside. ${ }^{15}$ In patients with cardiogenic shock, anterior T-wave inversions and ST elevation in leads aVR and V1 are associated with $\mathrm{PE}{ }^{12,18}$

In addition to the previously listed PoCUS findings, bedside diagnosis of $\mathrm{PE}$ is based on the visualization of a thrombus in the right heart as has been described. ${ }^{19}$ While this particular finding was not noted during resuscitation in our case, a subsequent review of our patient's bedside ultrasound images did reveal echogenic material in the right pulmonary artery (Figure 3). Clinically, the presence of a grossly abnormal right ventricular shape and function on PoCUS contributed significantly to our immediate treatment decisions. It is important to note that bedside echocardiography alone cannot safely rule out PE.,13 Nevertheless, the presence of particular findings on PoCUS in the context of a suspicious clinical presentation may strongly suggest the diagnosis. ${ }^{13}$ Other than a visible clot, specific echocardiographic findings reported in patients with $\mathrm{PE}$ are all consistent with right ventricular (RV) strain or overload: increased RV size, decreased RV function (both noted in our case), and tricuspid regurgitation. ${ }^{14}$ 


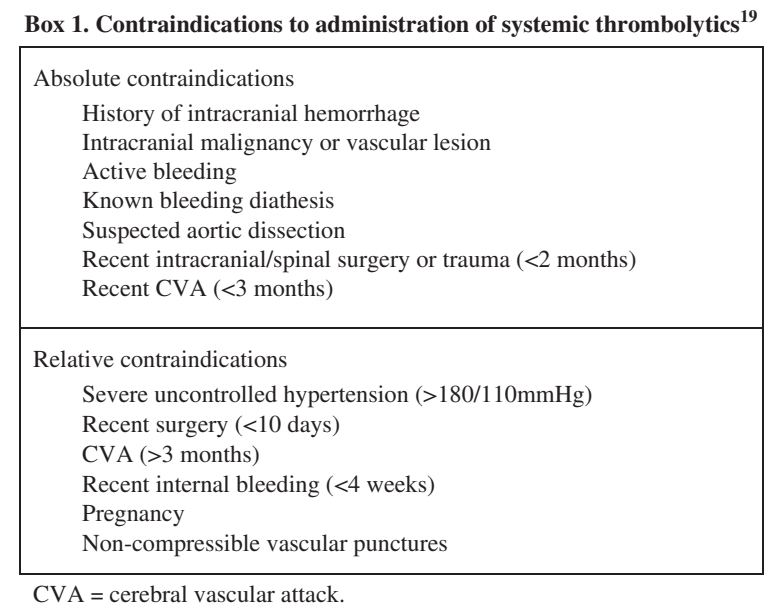

Figure 4. Contraindications to administration of systemic thrombolytics $^{19}$

\section{Treatment of high-risk PE}

Systemic thrombolysis is the standard of care in patients who present with high-risk PE, barring any contraindications (Figure 4). ${ }^{5,6,20-22}$ Existing literature has repeatedly confirmed a mortality benefit in patients with a high risk of $\mathrm{PE}^{23-26}$ The risk of death is reduced from $19.0 \%$ to $9.4 \%$ (absolute risk reduction [ARR] 9.6\%), as compared with anticoagulation alone. ${ }^{23}$ The most wellstudied and widely recommended systemic thrombolytic regimen for patients with $\mathrm{PE}$ who have a pulse is alteplase, $100 \mathrm{mg}$ IV over two hours. ${ }^{22,27}$ This regimen has been associated with earlier improvements in total pulmonary resistance, as compared with both older agents such as streptokinase (given over $12-24$ hours) ${ }^{28}$ or shorter infusions of alteplase of $0.6 \mathrm{mg} / \mathrm{kg}$ over 15 minutes. ${ }^{29} \mathrm{How}-$ ever, clinical outcomes and complication rates were similar across patient groups. ${ }^{28,29}$

Thrombolytic therapy carries a risk of major bleeding, including intracranial hemorrhage (ICH), with a number needed to harm of 11-176 quoted in the literature. ${ }^{7,23}$ In patients without absolute contraindications, the overall incidence of ICH after thrombolytic therapy was $0.9 \%-3 \% .4,30,31$ Approximately $20 \%$ of patients would experience a major hemorrhage ${ }^{4,32}$; gastrointestinal is most common, followed by retroperitoneal and intracranial. ${ }^{32}$ Predictors of major bleeding are relatively understudied but may include the need for vasoactive agents, malignancy, diabetes, recent bleeding, renal failure, dual anti-platelet therapy, and elevated international normalized ratio (INR). ${ }^{32}$ Bleeding risk is likely lower in those under age $65 .{ }^{7}$
Because of this bleeding risk, recent literature has focused on the safety and efficacy of reduced doses of thrombolytics for the treatment of PE. A randomized trial comparing $50 \mathrm{mg}$ per two hours of rt-PA with 100 $\mathrm{mg}$ per two hours of rt-PA demonstrated similar improvements in hemodynamic parameters, with significantly fewer bleeding episodes in the $50 \mathrm{mg}$ group. ${ }^{33}$ A subsequent prospective cohort of 98 patients with a PE (14 with a "severe PE") was treated with $50 \mathrm{mg}$ of IV alteplase over two hours, followed by unfractionated heparin and rivaroxaban, and no in-hospital deaths or bleeding episodes were reported. ${ }^{34}$ These dosing regimens may offer acceptable safety and efficacy in the treatment of patients with PE who have a pulse.

If systemic thrombolysis is contraindicated, alternative treatment options do exist. Depending on local resources and expertise, surgical thrombectomy or catheter-directed therapies may be appropriate. Recently published guidelines also recommend considering these modalities if systemic thrombosis has failed. ${ }^{5,22}$ The published risk of death with surgical thrombectomy is $5.3 \%-27 \%$, perhaps as high as $46 \%$ in the elderly. ${ }^{35-37}$ Published evidence supporting catheter-directed therapies in high-risk patients, including intravascular thrombolytics, suction, ultrasound-assisted, or rotational embolectomy, does not clearly favour one approach over others. ${ }^{38}$ The incidence of hemorrhagic and mechanical complications (e.g., dissection, perforation, and arteriovenous [AV] fistulization) is approximately $10 \% .{ }^{39}$ Published rates of ICH are lower than those observed with systemic lysis $(<1 \%) .{ }^{40}$ IVC filter placement is not routinely recommended for any patients with PE but may be considered in those with absolute contraindications to ongoing anticoagulation, including a major hemorrhage. ${ }^{5,22,41}$

In pulseless patients with $\mathrm{PE}$, proposed thrombolytic regimens have included:

- tenecteplase $50 \mathrm{mg}$ IV bolus ${ }^{42}$

- alteplase $~ 80 \mathrm{mg}$ IV bolus (dose chosen by treating physician $)^{1}$

- alteplase $50 \mathrm{mg}$ IV bolus, followed by $50 \mathrm{mg}$ 30 minutes later ${ }^{43}$

- alteplase $50 \mathrm{mg}$ IV bolus ${ }^{19}$

- alteplase $0.6-1.0 \mathrm{mg} / \mathrm{kg}$ (maximum dose $100 \mathrm{mg}$ ) IV bolus $^{44}$

As in the treatment of high-risk PE with a pulse, alteplase is the thrombolytic treatment of choice for PE 
in cardiac arrest. ${ }^{1,19,42-44}$ Its recommended shorter infusion time is more practical in critical illness and may promote more rapid clot lysis, as compared with the prolonged infusions of older agents such as streptokinase and urokinase. ${ }^{22,38,41}$ While case reports and case series can only provide limited quality and potentially biased evidence supporting specific drug regimens, the available literature does confirm the administration of rapid IV bolus alteplase (i.e., over minutes, rather than two hours) had encouraging outcomes in patients who experienced a cardiac arrest due to PE. Successful thrombolysis with tenecteplase, using weight-based dosing protocols extrapolated from the myocardial infarction literature, has also been reported in small numbers of cardiac arrest patients with suspected PE. ${ }^{10,11}$ The PEITHO study provided higher-level evidence for the use of bolus tenecteplase in PE, though this randomized controlled trial recruited only intermediate-risk patients. ${ }^{45}$ While its recommended administration as a rapid IV bolus makes it attractive for use during CPR, tenecteplase is not currently approved for this indication.

Traditionally, concern about hemorrhage precipitated by prolonged CPR has limited the use of systemic thrombolytics in arrested patients. ${ }^{44,46-47}$ Alteplase has the shortest half-life of all thrombolytics (4-10 minutes) and requires the presence of fibrin for activation; this may reduce the risk of bleeding complications by limiting the duration and extent of non-pathologic clot lysis. ${ }^{10}$ Furthermore, a retrospective review of 66 patients with confirmed PE and cardiac arrest, treated with systemic thrombolytics, did not find an increased incidence of bleeding if the duration of CPR exceeded 10 minutes. ${ }^{44}$ In patients who arrested with a high suspicion of $\mathrm{PE}$ and contraindication to thrombolysis (Figure 4), a clinician must decide whether to treat based on the individual patient's presentation, goals of care, and perceived risk of hemorrhage in each case. ${ }^{38}$

\section{CONCLUSION}

We administered a $100 \mathrm{mg}$ bolus of alteplase to a young, previously well patient with a high-risk PE. This treatment was associated with rapid ROSC, sustained hemodynamic stability, and neurologically intact survival after cardiac arrest, but also with a major hemorrhage requiring intervention. PoCUS may prove especially valuable in making a prompt working diagnosis in critically ill patients. Optimal thrombolytic agent and dosing in cardiac arrest remain unclear.
Competing interests: None declared.

\section{REFERENCES}

1. Er F, Nia AM, Gassanov $\mathrm{N}$, et al. Impact of rescuethrombolysis during cardiopulmonary resuscitation in patients with pulmonary embolism. PLoS One 2009;4(12):e8323.

2. Kasper W, Konstantinides S, Geibel A, et al. Management strategies and determinants of outcome in acute major pulmonary embolism: results of a multicenter registry. $7 \mathrm{Am}$ Coll Cardiol 1997;30(5):1165-71.

3. Moorjani N, Price S. Massive pulmonary embolism. Cardiol Clin 2013;31(4):503-18.

4. Goldhaber SZ, Visani L, De Rosa M. Acute pulmonary embolism: clinical outcomes in the International Cooperative Pulmonary Embolism Registry (ICOPER). Lancet 1999;353(9162):1386-9.

5. Konstantinides SV, Torbicki A, Agnelli G, et al. ESC Guidelines on the diagnosis and management of acute pulmonary embolism: the Task Force for the Diagnosis and Management of Acute Pulmonary Embolism of the European Society of Cardiology (ESC) Endorsed by the European Respiratory Society (ERS). Eur Heart 7 2014;35:3033-73.

6. Jaff MR, McMurtry MS, Archer SL, et al. American Heart Association Council on Cardiopulmonary, Critical Care, Perioperative and Resuscitation; American Heart Association Council on Peripheral Vascular Disease; American Heart Association Council on Arteriosclerosis, Thrombosis and Vascular Biology. Management of massive and submassive pulmonary embolism, iliofemoral deep vein thrombosis, and chronic thromboembolic pulmonary hypertension: a scientific statement from the American Heart Association. Circulation 2011;123(16):1788-830.

7. Chatterjee S, Chakraborty A, Weinberg I, et al. Thrombolysis for pulmonary embolism and risk of all-cause mortality, major bleeding, and intracranial hemorrhage: a meta-analysis. 7AMA 2014;311(23):2414-21.

8. Condliffe R, Elliot CA, Hughes RJ, et al. Management dilemmas in acute pulmonary embolism. Thorax 2014; 69(2):174-80.

9. Deng Y, He L, Yang J, Wang J. Serum D-dimer as an indicator of immediate mortality in patients with in-hospital cardiac arrest. Thromb Res 2016;143:161-5.

10. Logan JK, Pantle H, Huiras P, Bessman E, Bright L. Evidence-based diagnosis and thrombolytic treatment of cardiac arrest or periarrest due to suspected pulmonary embolism. Am 7 Emerg Med 2014;32(7):789-96.

11. Livaditis IG, Paraschos M, Dimopoulos K. Massive pulmonary embolism with ST elevation in leads V1-V3 and successful thrombolysis with tenecteplase. Heart 2004;90(7):e41.

12. Ferrari E, Imbert A, Chevalier T, et al. The ECG in pulmonary embolism. Predictive value of negative $\mathrm{T}$ waves in precordial leads-80 case reports. Chest 1997;111(3):537-43.

13. Squizzato A, Galli L, Gerdes VE. Point-of-care ultrasound in the diagnosis of pulmonary embolism. Crit Ultrasound 7 2015;7(1):7.

14. Come PC. Echocardiographic evaluation of pulmonary embolism and its response to therapeutic interventions. Chest 1992;101(4 Suppl):151S-162SS. 
15. Lin JF, Lin YC, Yang PL. A case of massive pulmonary embolism with ST elevation in leads V1-4. Circ 7 2009;73:1157-9.

16. Falterman TJ, Martinez JA, Daberkow D, Weiss LD. Pulmonary embolism with ST segment elevation in leads V1 to V4: case report and review of the literature regarding electrocardiographic changes in acute pulmonary embolism. 7 Emerg Med 2001;21(3):255-61.

17. Cheng TO. Mechanism of ST-elevation in precordial leads $\mathrm{V}(1)-\mathrm{V}(4)$ in acute pulmonary embolism. Int $\mathcal{7}$ Cardiol 2009;136(3):251-2.

18. Kukla P, McIntyre WF, Fijorek K, et al. Electrocardiographic abnormalities in patients with acute pulmonary embolism complicated by cardiogenic shock. Am 7 Emerg Med 2014;32(3):507-10.

19. Sharifi M, Berger C, Beeston P, et al. "PEAPETT" investigators. Pulseless electrical activity in pulmonary embolism treated with thrombolysis (from the "PEAPETT" study). Am $f$ Emerg Med 2016;34(10):1963-7, doi:10.1016/j. ajem.2016.094.

20. Moorjani N, Price S. Massive pulmonary embolism. Cardiol Clin 2013;31(4):503-18.

21. Konstantinides S, Goldhaber SZ. Pulmonary embolism: risk assessment and management. Eur Heart $\mathcal{f}$ 2012; 33(24):3014-22.

22. Kearon C, Akl EA, Comerota AJ, et al. Antithrombotic therapy for VTE disease: Antithrombotic Therapy and Prevention of Thrombosis, 9th ed: American College of Chest Physicians Evidence-Based Clinical Practice Guidelines. Chest 2012;141(2 Suppl):e419S-6S.

23. Wan S, Quinlan DJ, Agnelli G, Eikelboom JW. Thrombolysis compared with heparin for the initial treatment of pulmonary embolism: a meta-analysis of the randomized controlled trials. Circulation 2004;110(6):744-9.

24. Stein PD, Matta F. Thrombolytic therapy in unstable patients with acute pulmonary embolism: saves lives but underused. Am 7 Med 2012;125(5):465-70.

25. Jerjes-Sanchez C, Ramirez-Rivera A, de Lourdes García M, et al. Streptokinase and heparin versus heparin alone in massive pulmonary embolism: a randomized controlled trial. 7 Thromb Thrombolysis 1995;2(3):227-9.

26. Le Conte P, Huchet L, Trewick D, et al. Efficacy of alteplase thrombolysis for ED treatment of pulmonary embolism with shock. Am 7 Emerg Med 2003;21(5):438-40.

27. Lankeit M, Konstantinides S. Thrombolytic therapy for submassive pulmonary embolism. Best Pract Res Clin Haematol 2012;25(3):379-89.

28. Meneveau N, Schiele F, Metz D, et al. Comparative efficacy of a two-hour regimen of streptokinase versus alteplase in acute massive pulmonary embolism: immediate clinical and hemodynamic outcome and one-year follow-up. $7 \mathrm{Am}$ Coll Cardiol 1998;31(5):1057-63.

29. Sors H, Pacouret G, Azarian R, et al. Hemodynamic effects of bolus vs 2-h infusion of alteplase in acute massive pulmonary embolism. A randomized controlled multicenter trial. Chest 1994;106(3):712-7.

30. Stein PD, Matta F, Steinberger DS, Keyes DC. Intracerebral hemorrhage with thrombolytic therapy for acute pulmonary embolism. Am $\mathcal{Z}$ Med 2012;125(1):50-6.
31. Konstantinides S, Marder VJ. Thrombolysis in venous thromboembolism. In Hemostasis and thrombosis, (eds. Colman RW, Marder VJ, Clowes AW, et al.). Philadelphia: Lippincott Williams and Wilkins; 2006: 1317-29.

32. Fiumara K, Kucher N, Fanikos J, Goldhaber SZ. Predictors of major hemorrhage following fibrinolysis for acute pulmonary embolism. Am $\mathcal{F}$ Cardiol 2006;97(1):127.

33. Wang C, Zhai Z, Yang Y, et al.; China Venous Thromboembolism (VTE) Study Group. Efficacy and safety of low dose recombinant tissue-type plasminogen activator for the treatment of acute pulmonary thromboembolism: a randomized, multicenter, controlled trial. Chest 2010;137(2):254-62.

34. Sharifi M, Bay C, Schwartz F, Skrocki L. Safe-dose thrombolysis plus rivaroxaban for moderate and severe pulmonary embolism: drip, drug, and discharge. Clin Cardiol 2014;37(2):78-82.

35. Fukuda I, Taniguchi S, Fukui K, Minakawa M, Daitoku K, Suzuki Y. Improved outcome of surgical pulmonary embolectomy by aggressive intervention for critically ill patients. Ann Thorac Surg 2011;91(3):728-32.

36. Kilic A, Shah AS, Conte JV, Yuh DD. Nationwide outcomes of surgical embolectomy for acute pulmonary embolism. 7 Thorac Cardiovasc Surg 2013;145(2):373-7.

37. Stein PD, Matta F. Pulmonary embolectomy in elderly patients. Am 7 Med 2014;127(4):348-50.

38. Tapson VF. Thrombolytic therapy for acute pulmonary embolism. Semin Thromb Hemost 2013;39(4):452-8.

39. Kuo WT, Gould MK, Louie JD, et al. Catheter-directed therapy for the treatment of massive pulmonary embolism: systematic review and meta-analysis of modern techniques. f Vasc Interv Radiol 2009;20(11):1431-40.

40. Mostafa A, Briasoulis A, Telila T, Belgrave K, Grines C. Treatment of massive or submassive acute pulmonary embolism with catheter-directed thrombolysis. Am 7 Cardiol 2016;117(6):1014-20.

41. Walter RJ, Moores LK, Jiménez D. Pulmonary embolism: current and new treatment options. Curr Med Res Opin 2014;30(10):1975-89.

42. Perrott J, Henneberry RJ, Zed PJ. Thrombolytics for cardiac arrest: case report and systematic review of controlled trials. Ann Pharmacother. 2010;44(12):2007-13.

43. Ruiz-Bailén M, Aguayo-de-Hoyos E, Serrano-Córcoles MC, et al. Thrombolysis with recombinant tissue plasminogen activator during cardiopulmonary resuscitation in fulminant pulmonary embolism. A case series. Resuscitation 2001;51(1):97-101.

44. Janata K, Holzer M, Kürkciyan I, et al. Major bleeding complications in cardiopulmonary resuscitation: the place of thrombolytic therapy in cardiac arrest due to massive pulmonary embolism. Resuscitation 2003;57(1):49-55.

45. Meyer G, Vicaut E, Danays T, et al. PEITHO Investigators. Fibrinolysis for patients with intermediate-risk pulmonary embolism. N Engl 7 Med 2014;370(15):1402-11.

46. Li X, Fu QL, Jing XL, et al. A meta-analysis of cardiopulmonary resuscitation with and without the administration of thrombolytic agents. Resuscitation 2006;70(1):31-6.

47. Newman DH, Greenwald I, Callaway CW. Cardiac arrest and the role of thrombolytic agents. Ann Emerg Med 2000;35(5):472-80. 